\title{
Cryogenic TcBF-STEM Imaging of Vitrified Apoferritin with the Electron Microscope Pixel Array Detector
}

\author{
Yue Yu, Katherine Spoth, David Muller and Lena Kourkoutis \\ Cornell University, Ithaca, New York, United States
}

Cryo-transmission electron microscopy (cryo-TEM) has become a powerful tool for the investigation of biological ultrastructure. Compared to TEM, scanning TEM (STEM) is rarely used for biological systems because conventional STEM detectors discard portions of incident electron beam to form images making it difficult to study dose-sensitive materials. However, with the advent of pixelated direct detectors for STEM, which collect full convergent beam electron diffraction (CBED) pattern at each scan pixel, almost every incident electron can be collected. With a full 4D-STEM dataset, images can then be reconstructed after acquisition by integrating over any chosen detector geometry or by employing more complicated imaging methods. Tilt-corrected bright-field STEM (tcBF-STEM) [1], for example, has been shown to result in a five-fold increase in dose-efficiency compared to imaging with conventional bright field detectors [2]. The benefit of tcBF-STEM compared to standard EFTEM for imaging of thick biological samples such as whole $E$. coli cells has previously been demonstrated [1].

Here, we image frozen-hydrated horse spleen apoferritin using the Electron Microscope Pixel Array Detector (EMPAD), a direct detector optimized for STEM through its high dynamic range, single electron sensitivity and fast readout speed [3]. Apoferritin, a hollow, roughly spherical protein cage with an outer diameter of $12.5 \mathrm{~nm}$ and a molecular weight of $440 \mathrm{kDa}$, is often used for benchmarking of single particle cryo-EM setups. Purified apoferritin complexes were plunge frozen in liquid ethane and imaged at a defocus of -100 $\mathrm{nm}$ in an FEI Titan Themis equipped with a cryo-box operating at $300 \mathrm{kV}$.

In a conventional BF-STEM image (Fig. 1A), obtained by integrating the signal up to $1 / 3$ the probe convergence angle, $\alpha$, apoferritin complexes are visible, but the signal-to-noise SNR is low. To improve dose-efficiency we extended the detector "size" to $4 / 5 \alpha$ to produce an incoherent BF-STEM image (Fig. 1B). While the signal increases, microscopic details blur out due to defocus aberrations as off-axis detector pixels are included. To correct for such blurring, in tcBF-STEM each detector pixel is used as a coherent BF detector. Shifts between individual images can be quantified through cross-correlation (Fig. 2) and subsequently corrected for. Summing of all tilt-corrected images produces the tcBF-STEM image. The tilt-correction method can also provide an increase in real-space resolution of the final image compared to the original scan sampling. Redundant information in diffraction space is used to fill in information between real-space pixels in the scan. This information is coded in the image shifts determined to subpixel resolution by fitting the cross-correlation peak. The final upsampled tcBF-STEM image is produced by upsampling each image immediately prior to applying the shifts. The resolution limit is therefore not set by the acquisition scan pixel size. Here we upsampled the image by 4, reducing the pixel size from $7.98 \AA$ to $2.00 \AA$. Apoferritin complexes with high-SNR are clearly resolved in the final tcBF-STEM (Fig. $1 \mathrm{C})$.

As a key element of the tcBF-STEM method, the image shifts contain important physical information. Earlier work has demonstrated using such shifts to diagnose probe aberrations [4]. Figure 2A shows the shifts in $\mathrm{x}\left(\mathrm{S}_{\mathrm{x}}\right)$ and $\mathrm{y}\left(\mathrm{S}_{\mathrm{y}}\right)$ direction. The full $2 \mathrm{D}$ shift vector-field map is plotted as overlay on the average CBED pattern of the 4D dataset. The shifts point radially outwards, and the magnitude increases in radial 
direction which consistent with the defocus applied during the acquisition to enable upsampling and enhance phase contrast. Fitting of the shift matrix allows direct measurement of the aberration function.

The results presented here show that tcBF-STEM allows for high-SNR imaging of vitrified apoferritin complexes. Further improvements are expected with full field electron ptychography which makes use of all scattered electrons. [5]
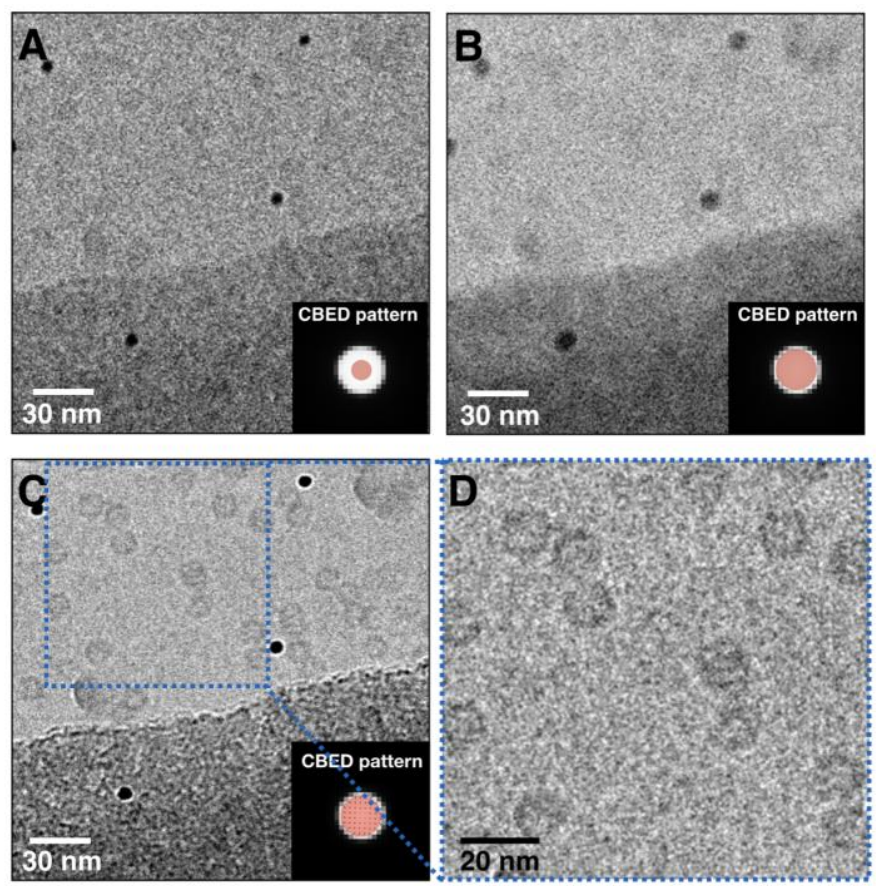

Figure 1. Cryo-STEM imaging of vitrified apoferritin with the electron microscope pixel array detector (EMPAD). Insets show the CBED pattern summed over all scan positions overlaid with red circles indicating the portion of CBED used for image formation. The convergence angle, $\alpha$, is $~ 2.9$ mrad. (A) Conventional BF-STEM image formed with electrons scattered up to $1 / 3 \alpha$. When electrons up to $4 / 5 \alpha$ are included an incoherent BF-STEM image obtained (B). (C) The tcBF-STEM image is the sum of cross-correlated images formed from each pixel in the central disk of the CBED pattern. Shifts of individual images are indicated as arrows. (C-D) Individual apoferritin particles embedded in vitrified ice are clearly resolved by cryogenic tcBF-STEM.
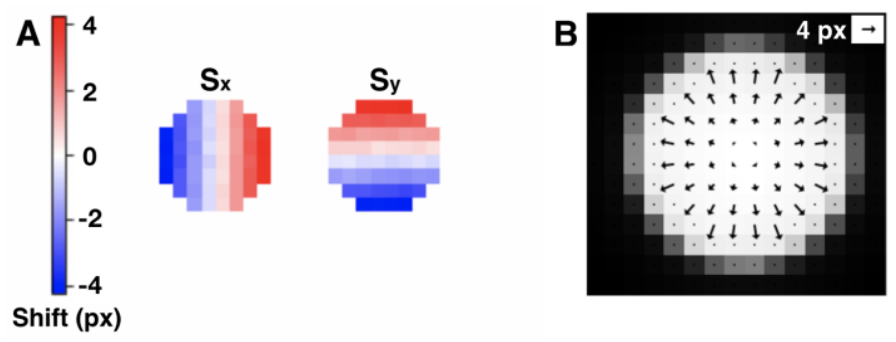

Figure 2. Quantification of image shifts for each image formed from a pixel in the central disk of the CBED pattern. (A) Image shifts, Sx and Sy, in the $\mathrm{x}$ and y direction, respectively. (B) Total shift for each image plotted as overlay on the average CBED pattern. The scale arrow in the top right corner indicates a 4-pixel image shift in the $+x$ direction. 


\section{References}

[1] K. A. Spoth, et al, Microscopy and Microanalysis 23 (2017), p. 804-805.

[2] K. A. Spoth, et al, Microscopy and Microanalysis 24 (2018), p. 876-877.

[3] M. W. Tate, et al, Microscopy and Microanalysis 22 (2016), p. 237-249.

[4] A. R. Lupini, et al, Journal of Microscopy 263 (2016), P. 43-50.

[5] Work supported through by the Packard foundation and NSF (DMR-1539918, DMR-1429155, DMR1719875). 